\title{
HIPERTENSIÓN ARTERIAL E INSUFICIENCIA RENAL CRÓNICA: REPERCUSIONES BUCALES, UNA REVISIÓN
}

\author{
${ }^{1}$ Martha Rebolledo Cobos, ${ }^{2}$ Ricardo Ibarra Kammerer \\ ${ }^{1}$ Estomatóloga y cirujana oral. Docente investigador de Patología, Semiología y Cirugía Oral, \\ director de grupo GIOUMEB. Universidad Metropolitana de Barranquilla, Colombia \\ ${ }^{2}$ Odontólogo, Universidad del Magdalena, Colombia
}

Autor responsable de correspondencia: Martha Rebolledo Cobos Correo electrónico: mrebolledo@unimetro.edu.co

\begin{abstract}
RESUMEN
Tanto la enfermedad cardiovascular como la enfermedad renal constituyen dos realidades fisiopatológicas de reconocimientos mortales crecientes en el ámbito mundial y de prioridades en materia de salud integral. En efecto, mientras que la hipertensión arterial (HTA) y la aterosclerosis son causas, cada vez más frecuentes, de nefropatía, este deterioro crónico de la función renal genera un estado vasculopático que facilita el desarrollo de lesiones del sistema cardiovascular, considerándose así, como enfermedades que van de la mano. Estos signos, no son aislados, a su vez se comportan como factores desencadenantes de afecciones bucodentales, como xerostomía, agrandamiento gingival, edema gingival, enfermedad periodontal, hemorragias petequiales, entre otras lesiones estomatológicas que empeoran el pronóstico de la enfermedad sistémica, afectando la calidad de vida del paciente y en diversas ocasiones dichas condiciones orales, según la gravedad, se comportan como factores etiológicos de enfermedad sistémica. Estas manifestaciones patológicas normalmente son vistas como de baja importancia por parte de los pacientes, debido a que sus prioridades corresponden a atender su situación sistémica. El propósito de esta revisión narrativa es describir las principales repercusiones bucales de cardiopatías y nefropatías, aportándole a la comunidad científica, académica y médico-odontológica conceptos actuales y elementales en la relación directa que poseen estas dos entidades sistémicas con la cavidad bucal. [Rebolledo M, Ibarra R. Hipertensión arterial e insuficiencia renal crónica: repercusiones bucales, una revisión. Ustasalud. 2015;14:53-58].
\end{abstract}

Palabras clave: Salud bucal, nefropatías, cardiopatías, hipertensión, falla renal.

\section{Hypertension and chronic renal insufficiency: Buccal Impact, a review}

\begin{abstract}
Both cardiovascular disease and kidney disease are two pathophysiological deadly realities of growing worldwide recognition and priorities for overall health. Indeed, while high blood pressure (hypertension) and atherosclerosis are becoming more common causes of kidney disease, this chronic deterioration of kidney function generates a vasculopátic state that facilitates the development of lesions of the cardiovascular system as well as diseases that are considered to the hand. These systemic conditions, they will not isolated, in turn behave as triggers of oral conditions, such as dry mouth, gingival enlargement, gingival edema, periodontal disease, petechial hemorrhages, including oral manifestations that worsen the prognosis of systemic disease, affecting quality of life of patients and on several occasions oral conditions such as gravity, act as etiological factors of systemic disease. These effects are usually cause for impairment by patients, because their priorities are to address systemic situation and is not cause for concern. The purpose of this narrative review is to describe the main impact of oral disease and kidney disease, bringing the basic direct relationship in the scientific, academic, medical and dental, current concepts and possess both systemic institutions with the oral cavity.
\end{abstract}

Keywords: Oral health, kidney disease, heart disease, hypertension, renal failure.

Recibido para publicación: 16 de noviembre de 2015. Aprobado para publicación: 10 de diciembre de 2015.

\section{INTRODUCCIÓN}

Las enfermedades cardiovasculares y renales, así como otras inmunosupresoras están entre las principales razones de muerte y morbilidad en el mundo, en la actualidad. Todo esto se relaciona con el cambio en el estilo de vida de la población. Estas condiciones vienen de la mano con nuevos hábitos que han afectado, poco a poco, el cuerpo humano y perjudicado la salud. Entre las razones para hacer un seguimiento de estas patologías se encuentra la repercusión que ellas conllevan sobre los otros sistemas y órganos. Por ejemplo, los efectos que se manifiestan en la cavidad bucal por el consumo de antihipertensivos, o en la misma elevación de la presión en hipertensos; generando lesiones o patologías que suelen ser asintomáticas. Por otro lado, las cardiopatías afectan directamente a los riñones, generando una mala función en estos, lo que lleva a un deficiente funcionamiento de otros órganos y sistemas enlazados a los órganos renales; en cuanto al daño que producen sobre la boca, las patologías renales pueden desencadenar pérdida ósea y palidez de las mucosas. Teniendo en cuenta esta problemá- 
tica, el objetivo de la presente revisión narrativa es describir las principales características bucales de pacientes con diagnóstico de hipertensión arterial e insuficiencia renal crónica.

\section{LAS ENFERMEDADES CARDIOVASCULARES Y LA BOCA}

Las enfermedades cardiovasculares (ECV) constituyen en la actualidad una causa frecuente de incapacidad y muerte prematura en el mundo; se calcula que en 2011 murieron por esta causa 17,5 millones de personas, lo cual representa un 31\% de todas las muertes registradas en el mundo. De estas muertes, 7,4 millones se debieron a la cardiopatía coronaria, y 6,7 millones, a los AVC (accidentes vascular-cerebral) $)^{1,2}$. Esto se debe en gran medida a cuatro factores de riesgo comportamentales que se han afianzado de forma generalizada como parte de la transición económica, los rápidos procesos de urbanización y los modos de vida del siglo XXI: el consumo de tabaco, las dietas malsanas, la inactividad física y el uso excesivo del alcohol ${ }^{1,3}$. La cardiopatía isquémica es la afección vascular de mayor letalidad, responsable de aproximadamente el 14\% de todas las muertes en todo el mundo (cerca de 7,5 millones de personas cada año); esto muestra que se debe tener mayor atención y hacer campañas de promoción y prevención sobre esta enfermedad y su importancia en el ámbito mundial ${ }^{1}$.

En cuanto a la patogenia de la enfermedad cardiovascular, son numerosas las incógnitas que quedan por resolver y solo se ha establecido que el tabaquismo y ciertas enfermedades como Diabetes Mellitus (DM), Hipertensión Arterial (HTA) e hipercolesterolemia y hábitos del paciente, entre otros, favorecen el desarrollo de la ateroesclerosis; los cuales constituyen los denominados factores de riesgo vascular ${ }^{4-8}$.

Estudios experimentales, epidemiológicos y otros han podido demostrar una clara evidencia de la asociación multicausal entre estos factores de riesgo, aunque el impacto es individual, está bien establecido y su prevención mejora la predicción del riesgo cardiovascular ${ }^{4-6,9}$. En décadas pasadas se ha apreciado un énfasis creciente en el tratamiento del riesgo cardiovascular global, el cual requiere la evaluación y el tratamiento de los múltiples factores de riesgo ya descritos, se debe tener en cuenta que estudios epidemiológicos a gran escala han evidenciado que los factores de riesgo tienen un efecto sinérgico, más que aditivo, sobre el riesgo cardiovascular total ${ }^{9,10}$.

Así como las enfermedades cardiovasculares afectan diversos órganos y sistemas, lo que conlleva la descompensación sistémica y disminución de la sobrevida del paciente, también tienen relación directa e indirecta con la presencia de múltiples enfermedades estomatológicas de características variables y de evolución diferente, que comúnmente son omitidas por el médico tratante, lo que genera que la interconsulta con el odontólogo o estomatólogo sea irrelevante 4 . Algunas de estas patologías bucales son desencadenadas, ya sea por la propia enfermedad sistémica de base o por las terapéuticas farmacológicas empleadas para el manejo paliativo del estado sistémico ${ }^{4,11,12}$.

Autores como Aguilar y colaboradores ${ }^{11}$ reportan que las enfermedades vasculares presentan repercusiones sobre el periodonto; en su fisiología y anatomía. Por ejemplo, la HTA tiene un efecto sobre la mucosa bucal, lo que crea en ella pequeñas extravasaciones denominadas petequias o hemorragias petequiales, considerándose en la actualidad por múltiples investigadores como la única manifestación bucal de la HTA, cuya razón de ser, es atribuible al aumento severo y súbito de la presión arterial, aunque también debe considerarse su aparición en condiciones secundarias, como al empleo de antihipertensivos $^{13}$ (Figura 1).

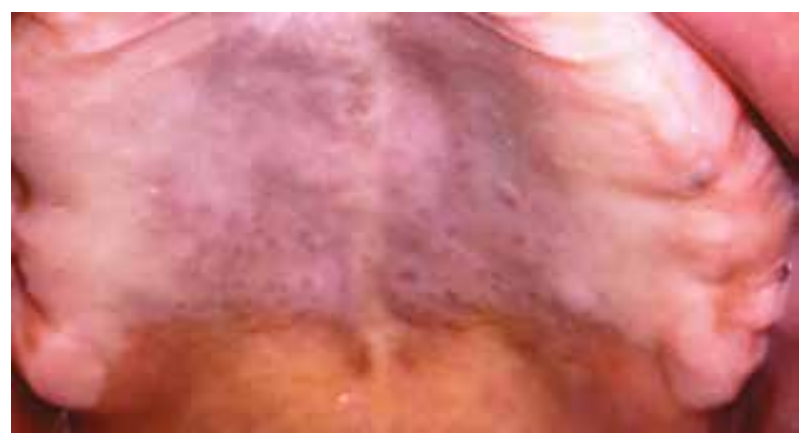

Figura 1. Múltiples puntos de color rojo violáceo en mucosa, de tamaños variables ubicados en tercio medio y posterior del paladar duro compatible con petequias, presentes en la HTA.

Los pacientes con riesgo cardiovascular se deben tratar con terapias antibióticas de soporte sistémico (profilaxis antibióticas), para evitar que microorganismos presentes en patologías de la cavidad bucal se transfieran al torrente sanguíneo y formen colonias en los vasos o en el mismo corazón, afección denominada endocarditis infecciosa $(\mathrm{EI})^{11,14,15}$. El insuficiente cuidado de las enfermedades periodontales y bucales en general, puede conllevar al paciente a un estado alarmante con formación de colonias bacterianas en el corazón, estos argumentos se traducen como que las enfermedades del corazón en ocasiones pueden ser originadas por alteraciones bucodentales no manejadas, sobre todo del tipo infeccioso ${ }^{11,14,15}$.

Desde el punto de vista bucal, las complicaciones potenciales que atentan contra la vida pueden ser 
prevenidas al realizar el tratamiento bucal profiláctico en pacientes con HTA severa, pero la verdadera tarea consiste en hacer que el paciente asista con el médico general para que se haga una valoración y así se obtenga un diagnóstico y así mismo se le dé tratamiento ${ }^{13}$.

El autor Jiménez-Beato comenta que la utilización de diversos fármacos para atender las enfermedades cardiovasculares, incide directamente en la cavidad bucal, lo cual genera manifestaciones en los diferentes tejidos que se encuentran en la boca, lo que lleva a que se afecten directamente los órganos dentales ${ }^{14,15}$.

Dentro de la utilización de inhibidores de la enzima convertidora de angiotensina (IECA) y su efecto sobre el complejo renina-angiotensina-aldosterona crean enfermedades en la cavidad bucal que se vuelven incidentes y prevalentes en la utilización de otros antihipertensivos (xerostomía, hemorragias, enfermedad periodontal) lo que implica la exposición de cuellos o zonas cervicales dentales, agrandamientos y edemas gingivales ${ }^{16}$. Los bloqueadores de canales de sodio tienen repercusión sobre la contextura de la saliva y las encías, entonces la utilización de diferentes fármacos para tratar la HTA, cardiopatías y nefropatías son sinérgicos para las afecciones bucales que se presenten ${ }^{11,12,16}$ (Tabla 1). PONER aquí la tabla 1

Nuevas formas de tratamientos no farmacológicos están siendo investigadas, para así disminuir los efectos que tiene el alto consumo de fármacos sobre los diferentes tejidos y sistemas en los pacientes. Walaszek ofrece una nueva forma en su investigación sobre los masajes clásicos, aplicados a un grupo de mujeres entre los 60 y 68 años de edad; encontrando una disminución de la presión arterial, y ofrece este método como una forma coadyuvante para hacer frente a la utilización de fármacos y, además, bajar la repercusión de los medicamentos sobre los demás sistemas y la boca, asimismo mejora el estado hepático del paciente ${ }^{17}$. Estos masajes están siendo aplicados en conjunto con medicamentos en dosis menores, logrando mantener y mejorar el manejo de la presión arterial en ellos ${ }^{17}$.

\section{Insuficiencia renal crónica y la boca}

La insuficiencia renal crónica (IRC) se define como la pérdida gradual y progresiva de la capacidad de los riñones para depurar solutos, concentrar la orina y conservar los electrolitos con el consiguiente deterioro de la función renal, por tanto ocurre la retención de diversos productos de desechos del metabolismo como la urea, nitrógeno ureico y creatinina, estado denominado azoemia ${ }^{19,20}$. Esta afección metabólica es considerada como uno de los principales factores etiológicos de ECV, diversos estudios manifiestan que entre el $40 \%$ y el $75 \%$ de los pacientes que comienzan los programas de diálisis y hemodiálisis, tienen $\mathrm{ECV}^{20-22}$. Esta condición a su vez, es responsable del $44 \%$ de las muertes de pacientes en esta situación y constituye, teniendo en cuenta la edad y el sexo, la causa más importante de morbilidad cardiovascular y de mortalidad tota $^{21}$. Estos cuadros clínicos, antes mencionados, crean el síndrome de insuficiencia cardíaca crónica; el cual se caracteriza por una limitación progresiva de la actividad física, retención de sal y agua, activación neurohormonal, alteraciones estructurales y funcionales cardiacas ${ }^{21-27}$.

Estudios realizados por Rebolledo, Carmona y col, en comparación con los de Belazelkovska y col, manifiestan que ningún paciente con IRC, participante de sus investigaciones, se encontró sano bucalmente durante las fases de hemodiálisis; así mismo, reportaron que durante el examen semiológico intraoral ${ }^{21}$ la prevalencia de lesiones orales fue encabezada por caries en un $83,9 \%$, seguida de la presencia de placa bacteriana en un $72,3 \%$, restos radiculares en un $64,2 \%$ y cálculo en un $61,6 \%$, igualmente otras lesiones como; xerostomía, pseudoplaca en lengua, palidez de las mucosas, movilidad de órganos dentales, atrofia, sabor, olor urémico y gingivorragia ${ }^{21,22}$. Así como la mayoría de cardiopatías, también la IRC repercute en la salud bucal y es directamente proporcional al estado de salud oral ${ }^{21,22}$.

Del mismo modo, otros investigadores afirman que el $90 \%$ de los pacientes con IRC sufren signos y síntomas bucales, que afectan tanto al hueso, como lo es la osteodistrófia renal; que es sinérgico y paralelo a un hiperparatiroidismo que afecta la conformación del hueso, dependiendo de la causa ${ }^{23}$. Una de las zonas que presenta mayor afectación es el maxilar y hueso alveolar, afectando así la salud bucal del paciente, volviéndolo propenso a la fractura y a la reabsorción, de igual manera a los tejidos blandos, sin embargo, estos no son patognomónicos ni determinantes de la enfermedad renal, como sí lo son: la disgeusia y cacogeusia que resulta del aumento en la concentración de urea en la saliva debido a bacterias que la metabolizan y transforman en amoniaco; esto provoca en algunos pacientes sensación de macroglosia ${ }^{11,12,16,19,23}$.

Un estudio realizado por Espitia y cols ${ }^{18}$ demostró que afecciones como la Xerostomía, son patognomónicas y están directamente relacionadas con IRC en hemodiálisis a consecuencia de la restricción en la ingesta de líquidos, así mismo, otros efectos secundarios se hacen presentes, inherentes a los 
Tabla 1. Lesiones estomatológicas más frecuentes de la HTA e IRC con relación al uso de medicamentos y condición sistemica ${ }^{11,12,16,17,36}$

\section{MANIFESTACIÓN ORAL}

Xerostomía

Petequias

Palidez de las mucosas

Periodontitis

Hiposalivación

Candidiasis

Úlceras aftosas

Caries

Aliento amoniacal

Reacciones linquenoides

Otras autoinmunes

\section{DEFINICIÓN Y SÍNTOMAS}

Síntoma oral, sugestivo, incómodo y potencialmente perjudicial que por lo general es causado por la disminución de la secreción salival. Bastante frecuente en la población de edad avanzada y con alto consumo de medicamentos (diuréticos y los IECA).

Pequeñas extravasaciones puntiformes y asintomáticas, debido al aumento severo y súbito de la presión arterial, pero su aparición puede identificarse en condiciones secundarias como al empleo de antihipertensivos.

Por la afectación directa que ejercen los tratamientos farmacológicos de la HTA e IRC sobre capilares y mucosa, disminución de la hemoglobina por déficit de eritropoyetina a nivel renal.

Afectación generada por el bajo pH (diuréticos) y la afectación al sistema inmune en la quimiotaxis.

Generada por afectación directa sobre las glándulas salivales en el consumo de medicamentos como diuréticos como IECA y por la restricción de la ingesta de líquidos.

Se hace presente en la afectación directa del sistema inmune y la baja producción de saliva que directamente reduce el $\mathrm{pH}$ oral favorecedor de la colonización fúngica.

Generadas por diversos mecanismos y relacionadas con el consumo de IECA así como la inmunosupresión generada por las condiciones sistémicas.

Generada por el bajo pH en la disminución de la salivación, IRC.

Se genera en la restricción de la ingesta de líquidos; y si a eso se suman los daños que pueden tener en las glándulas salivales, debido a los altos niveles de urea que presentan cuando no están controlados.

Generalmente en mucosa bucal, relacionadas con el consumo de tiazidas, propanol.

Relacionadas sus ocasionales apariciones con anticuerpos que atacan la membrana basal y que por activación del complemento separan la matriz del epitelio y tejido conectivo en el uso crónico de IECA.

medicamentos (fundamentalmente fármacos antihipertensivos) como; alteración de las glándulas salivales y respiración bucal, aumentando el riesgo de sufrir enfermedad periodontal, también otras manifestaciones orales como sangrado gingival, inflamación gingival, hiperplasia gingival, erosión dental, entre otras, se hacen presente durante estos estados sistémicos ${ }^{11,12,16,18,19}$ (Figura 2).

Hamissi y $\operatorname{cols}^{26}$ realizaron un estudio con 180 pacientes en Irán, el cual arrojó prevalencia de alteraciones orales en un $100 \%$ de ellos, siendo más comunes el cálculo dental, índice COP elevado y placa bacteriana con gingivorragia. Así como estos autores lo describen, Craig et al. ${ }^{27}$ y Dumitrescu et $\mathrm{al}^{28}$ en estudios independientes reportan que existe un mayor riesgo para el deterioro de estructuras dentales y alto índice de enfermedad periodontal en los pacientes con IRC, acompañado de ansiedad, estrés y depresión ${ }^{19,28}$.

Diversos autores han evidenciado una directa relación existente entre la IRC y la ECV, así como los efectos directos con el estado de salud en el pacien- te a nivel sistémico general; las secuelas que generan la enfermedad renal y la enfermedad cardiaca sobre la piel, la mucosa y otros tejidos, esto debe ser un signo para tomar medidas y afrontar de la mejor manera las afectaciones que se presentan en la cavidad bucal ${ }^{18,30-32-34}$. Al primer signo de estas repercusiones sobre los diferentes tejidos, sistemas y en la cavidad bucal, por parte de las ICR y ECV y el manejo de antihipertensivos debe ser observaba, reportada y manejada por el odontólogo o estomatólogo con interconsulta con el médico internista, para llevar un control sobre la severidad de estas sobre la cavidad bucal ${ }^{35-38}$.

\section{CONCLUSIONES}

Los resultados arrojados en esta revisión narrativa evidencian que la enfermedad cardiovascular y la enfermedad renal tienen una relación entre sí; lo que genera patologías entre órganos incluyendo el sistema estomatognático.

Entre las manifestaciones que en la cavidad bucal se presentan por la HTA; se encuentran las extra- 
vasaciones sanguíneas conocidas como petequias, estas son las de mayor presencia, pero, si a eso se le agrega el uso de antihipertensivos pueden aparecer agrandamientos gingivales, sangrado gingival, xerostomía, entre otros. Los pacientes que presenten ECV e IRC tienen mayor probabilidad de tener lesiones bucales asociadas a las patologías sistémicas o a la ingesta continua de medicamentos para su control. Las repercusiones bucales con mayor ocurrencia y más evidentes son la xerostomía, palidez en mucosa. Y si al mismo tiempo padece las dos enfermedades (ECV, IRC) y presenta un manejo farmacológico para su control, las afectaciones orales son mayores.

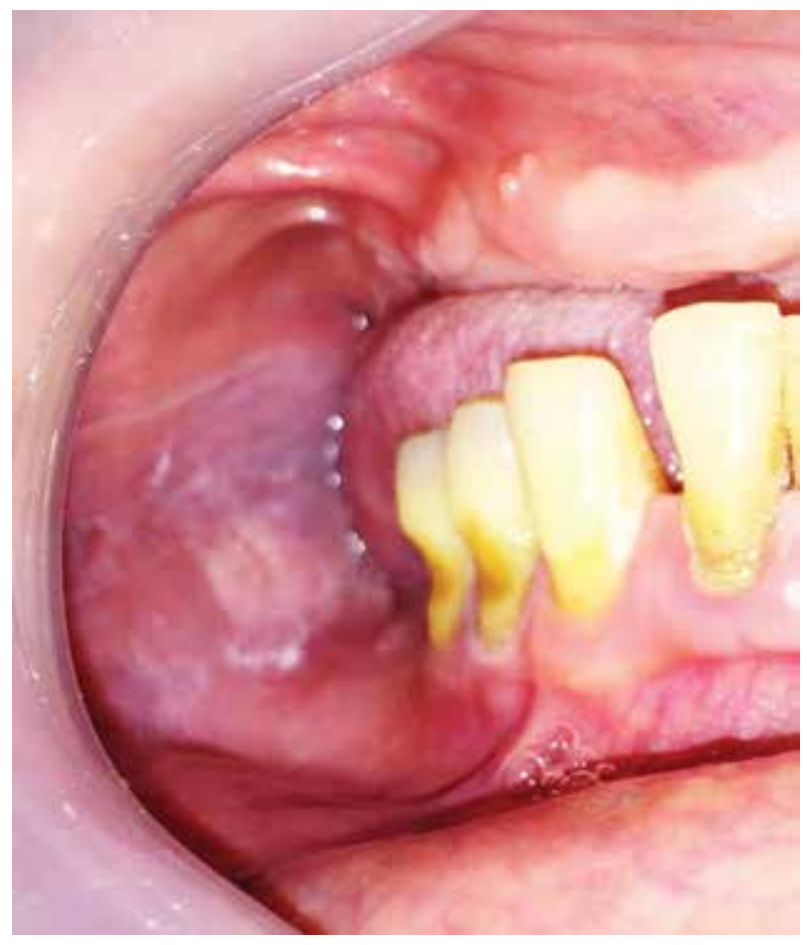

Figura 2. Se evidencia en mucosa yugal tercio posterior, placa blanquecina definida asociada a leucoedema que puede estar presente en la diabetes mellitus y en tercio cervical de órganos dentales se evidencia retracción gingival, la cual puede estar presente en la enfermedad renal o en el manejo de antihipertensivos.

Esto nos muestra que la IRC y ECV tienen no solo una repercusión sobre otros sistemas, sino también sobre la cavidad bucal; complicando los tratamientos dentales y la recuperación postratamientos odontológicos sobre el paciente. Estas consecuencias pueden empeorar y desencadenar patologías bucales más complicadas y afectar recíprocamente a los diferentes sistemas.

Por ende, en pacientes con cardiopatías o nefropatías y que estén bajo tratamiento farmacológico se recomienda unas visitas regulares al odontólogo o estomatólogo para llevar un control de la evolución en la cavidad bucal y las manifestaciones de estas enfermedades sobre el periodonto, la mucosa y los órganos dentales; para asimismo tener un mejor manejo, tomar medidas preventivas, y así evitar la complicación en la calidad de vida del paciente.

El manejo interdisciplinario da un apoyo al odontólogo sobre los medicamentos que afectan la salud bucal, y así implementar o crear nuevos protocolos de manejo para cada enfermedad que presenten los pacientes y se relacione directamente con estas afecciones. El uso de los diferentes fármacos para tratar las enfermedades afectan de forma directa e indirecta la cavidad bucal, por eso, la constante vigilancia y seguimiento que debe tener el odontólogo o estomatólogo con el médico internista, para verificar si es posible mejorar la terapia farmacología con una que no repercuta negativamente en la cavidad bucal.

\section{REFERENCIA}

1. World Health Organization. Global status report on non communicable diseases 2011. Acceso: 20 de abril de 2016. Recuperado a partir de: http://www.who.int/nmh/publications/ncd_report2010/es/

2. Fierro MG, Toaza AR. Hipertensión arterial crónica que se complica con insuficiencia cardíaca congestiva. UTA, Facultad Ciencias de la Salud, Carrera de Medicina. [serie en internet]. [citado 19 Abr 2016]. Recuperado a partir de: http://repositorio.uta.edu.ec/jspui/handle/123456789/9462

3. Sosa Liprandi MI, Racki M, Khoury M, Villareal R, Cestari G, Mele E et al. Crisis económico-financieras en la Argentina: ¿un nuevo factor de riesgo de mortalidad cardiovascular? Rev. argent. cardiol. [Internet]. $2012 \mathrm{Abr}$ [citado 2016 Abr 19]; 80(2):137-144. Recuperado a partir de: http://www.scielo.org.ar/scielo.php?script=sci arttext\&pid $=$ S1850-37482012000200007\&lng $=$ es

4. Barrett, Bhambri, Robbins. Uptitrating amlodipine significantly reduces blood pressure in diabetic patients with hypertension: A retrospective, pooled analysis. Vascular Health and Risk Management. 2014;10:651-9.

5. Salinas Botrán A, Ramos Rincón JM, de Górgolas Hernández-Mora M. Enfermedad cardiovascular: una visión desde la salud global. Elsevier. 2013;141(5):210-6.

6. Sellén Crombet J, Sellén Sanchén E, Barroso Pacheco L, Sellén Sánchez S. Evaluación y diagnóstico de la hipertensión arterial. Rev Cubana Invest Bioméd [Internet]. 2009 Mar [Acceso 2016 Abr 19];28(1). Recuperado a partir de: http://scielo.sld.cu/scielo.php?script $=$ sci arttext\&pid $=$ S0864-03002009000100001\&lng $=$ es.

7. Chan, Pang et al. Elevated lipoprotein (a), hypertension and renal insufficiency as predictors of coronary artery disease in patients with genetically confirmed heterozygous familial hypercholesterolemia. Int J Cardiol. Dec. 2015;15;201:633-8. doi: 10.1016/j.ijcard.2015.08.146. Epub 2015 Aug 21.

8. Kanai T, Krum H. New treatment for old disease: Management of resistant hypertension by oercutaneous renal sympathetic denervation. Rev Esp de Cardiol. 2013;66(9):734-740.

9. Tejerina Lobo, Cuesta Frechoso et al. ¿Existe relación entre enfermedad cardiovascular y periodontitis? Avances en Periodoncia 2003;15(3):113-9. 
10. Mur Martí et al. Prevalencia de enfermedad renal crónica en pacientes con diabetes tipo 2 mediante determinación del filtrado glomerular y su relación con el riesgo cardiovascular. Medicina Clínica. 2013;140(9):395-400.

11. Aguilar et al. Manifestaciones bucales en pacientes hipertensos bajo tratamiento antihipertensivo. Arch Inv Mat Inf. 2009;I(2):90-94.

12. Cedeño, Rivas, Tuliano. Manejo odontológico del paciente con enfermedad renal crónica terminal: Revisión Bibliográfica. Acta Odont Ven. 2013;51(1). Recuperado a partir de: http://www.actaodontologica.com/ediciones/2013/1/ art25.asp

13. Vega AJ, Guimará MM, Vega AL. Riesgo cardiovascular, una herramienta útil para la prevención de las enfermedades cardiovasculares. Rev Cubana Med Gen Integr. 2011;27(1):91-97.

14. Jiménez-Beato G, Machuca-Portillo G. Heart and periodontal diseases: Does evidence exist of association? Med Oral Patol Oral Cir Bucal. May-Jul 2005;10(3):215-20.

15. Liliana, Nicolosi et al. La enfermedad periodontal como factor de riesgo en la cardiopatía isquémica. Rev Arg Cardiol. 2003;71(4)250-255.

16. Orellana Centeno et al. Educación y cuidado estomatológico de pacientes infantiles con insuficiencia renal en pacientes de Soledad de Graciano Sánchez, San Luis Potosí. Rev Nal Odonto. 2014;8(15):57-62.

17. Walaszek R. Impact of classic massage on blood pressure in patients with clinically diagnosed hypertension. J Tradit Chin Med. 2015;35(4):396-401.

18. Espitia, Carbonell, Carmona. Hallazgos estomatológicos relacionados con insuficiencia renal crónica. Estudio de cohorte. Rev Duazary. 2006;2:95-103.

19. Lecca Rojas, Meza Mauricio, Ríos Villasis. Manifestaciones bucales en pacientes con insuficiencia renal crónica en hemodiálisis. Rev Estomatol Herediana. 2014;24(3):147-154.

20. Locatelli, Bommer et al. Cardiovascular disease determinants in chronic renal failure: clinical approach and treatment. Nephrol Dial Transplant. 2001;16:459-468.

21. Rebolledo CM, Carmona LM, Carbonell MZ. Salud oral en pacientes con insuficiencia renal crónica hemodializados después de la aplicación de un protocolo estomatológico. Av Odontoestomatol. 2012;28(2):77-87.

22. Belazelkovska et al. Oral clinical findings in patients with chronic renal failure. Balk J Stom. 2013;17(1):37-43.

23. Malluche HH, Davenport DL, Cantor T, Monier-Faugere MC. Bone mineral density and serum biochemical predictors of bone loss in patients with CKD on dialysis. Clin J Am Soc Nephrol. 2014;9(7):1254-1262.

24. Dávila Spinetti DF, Donis JH, González M, Sánchez F. Fisiopatología, diagnóstico y tratamiento médico de la insuficiencia cardiaca congestiva. Rev. Venez. Endocrinol. Metab. [Internet]. [Acceso 2016 Abr 20]. Dic. 2010;8(3):88-98. Recuperado a partir de: http://www.scielo.org.ve/scielo. php?script $=$ sci_arttext\&pid $=$ S16903110201000030000 $1 \& \operatorname{lng}=$ es

25. European Society of Hypertension - European Society of Cardiology guidelines for management of arterial hypertension. J Hypertens: 2003;21:1011-1053.
26. Hamissi, Porsamimi, Naseh, Mosalaei. Higiene bucal y el estado periodontal de los pacientes hemodializados con insuficiencia renal crónica en Qazvin, Irán. Este Afr Salud Publican J. 2009;6(1):108-11.

27. Lovera K, Delgado E, Bereni L, Gay C. El paciente con insuficiencia renal en la práctica odontológica. RCOE. 2000;5(5):521-531.

28. Craig RG, Kotanko P. Periodontitis and the endstage renal disease patient receiving hemodialysis maintenance therapy. Compend Contin Educ Dent. 2009;30(8):544-552.

29. Dumitrescu AL, Gârneapã L, Guzun O. Anxiety, stress, depression, oral health status and behaviours in Romanian hemodialysis patients. Rom J Intern Med. 2009;47(2):161168.

30. Cirera Segura F, Pérez Baena A, Sánchez Flores N, Martín Espejo JL. El control de la presión arterial en la consulta de enfermedad renal crónica avanzada. Enferm Nefrol. 2014;17(1):35-38.

31. Castro Lara J, Ibero Sagastibelza I, Bascones Martínez A. ¿Es la enfermedad periodontal un factor de riesgo para las enfermedades cardiovasculares? (II): Modelo biológico. Avances en Periodoncia [revista en la Internet]. [Acceso 2016 Abr 20] Dic. 2001;13(3):33-44. Recuperado a partir de: http://scielo.isciii.es/scielo.php?script $=$ sci arttext\&pid $=$ S1699-65852001000300005\&lng $=$ es.

32. Kaplan I1, Zuk-Paz L, Wolff A. Association between salivary flow rates, oral symptoms, and oral mucosal status. Oral Surg Oral Med Oral Pathol Oral Radiol Endod. 2008;106(2):235-241.

33. Robles-Méndez JC, Vázquez-Martínez O, Ocampo-Candiani J. Skin manifestations of chronic kidney disease Actas Dermo-Sifiliográficas, 2015;106(8):609-622.

34. Alberto, Zayas, Fragoso, Cuairan, Hernández. Manejo estomatológico en pacientes con insuficiencia renal crónica: presentación de caso. Rev Odont Mex. 2009;13(3):171176.

35. Scully. Drugs effects on salivary glands: Dry mouth. Oral Dis. Jul. 2003;9(4):165-76.

36. Nirmal Raj et al. Oral manifestations of chronic renal failure and its management. Review Article. J Res Adv Dent 2015;4(12):7-13.

37. Muñoz E, Restrepo C, Chacón J. Caracterización en salud oral y hábitos de higiene oral en pacientes con enfermedad renal crónica. Act Med Col. 2011;36(4):173-180.

38. Díaz A, Arévalo L, Fonseca M. Agrandamiento gingival inducido por nifedipina: Reporte de un caso. Rev Duazary. 2009;6(1):51-55.

\section{Correo electrónico de los autores}

Martha Rebolledo Cobos: mrebolledo@unimetro.edu.co Ricardo Ibarra Kammerer:rikad_185@hotmail.com 